\title{
Overall performance of a centrifugal vaned diffuser Pump for different flow rates, experimental and numerical comparisons
}

\author{
Taha El Amine TERKI HASSAINE, Abdelali SEDDINI, Hamid BOUCHELKIA \\ Department of hydraulic, EOLE Laboratory \\ University of Tlemcen, \\ BP 230, 13000, Tlemcen,
}

\section{ALGERIA}

\begin{abstract}
The article presents the analysis of the interactions between the impeller and the vaned diffuser of a radial flow pump. The tests were carried out on the so-called SHF impeller, coupled with a vaned diffuser, and working with air. The particularity of this machine is that the diffuser design flow rate corresponds to $80 \%$ of the impeller one. All experimental works were performed at the Fluid Mechanics Laboratory in ENSAM, Lille, France. Investigations have been made for five different flow rates. Global performances of the machine are evaluated thanks to pressure measurements and averaged velocities obtain with a three hole probe, at nine angular positions at diffuser inlet and outlet just as five radial positions in a middle section of a blade-to-blade passage. A post-processing procedure, based on statistical tools, was applied to the experimental results in order to reach a better understanding of the phenomena. In another approach, a numerical simulation of the flow inside the pump, for eight different relative angular positions of the diffuser relative to the impeller (Frozen rotor) was performed by the STAR-CCM+ software. The experimental results were compared to numerical data obtained with the help of STAR-CCM+ computer code.
\end{abstract}

Keywords: Centrifugal pump, STAR-CCM+, Frozen Rotor, SHF pump, Vaned diffuser

Received: November 26, 2019. Revised: April 20, 2020. Accepted: April 29, 2020. Published: May 19, 2020.

Nomenclature

\begin{tabular}{|ll|ll|}
\hline$N$ & rotation speed & $\mathrm{Q}_{\mathrm{d}}$ & design flow rate \\
$P$ & pressure & $Z_{i}$ & number of impeller blades \\
$\Delta \mathrm{P}_{\mathrm{s}}$ & static pressure & $Z_{d}$ & number of diffuser blades \\
$p_{t}$ & total pressure & $\rho$ & air density \\
$Q_{*}$ & volume flow rate in impeller & 1 & impeller inlet \\
$Q^{*}$ & $\mathrm{Q} / \mathrm{Q}_{\mathrm{d}}$ & 2 & impeller outlet \\
$Q_{n}$ & nominal volume flow rate & 3 & diffuser inlet \\
$U_{2}$ & rotational velocity at impeller outlet & 4 & diffuser outlet \\
\hline
\end{tabular}

\section{Introduction}

Interaction between rotating and stationary parts of a turbo machine is one of the main sources of unsteadiness, noise and vibration of this kind of machine, the results of which may affect efficiency in design and off design conditions. This requires a better understanding of unsteady effects that must be considered by measuring the local flow field characteristics both in the relative and absolute frame and so, inside the rotating impeller blades and the diffuser ones as well. The experimental data are increasingly needed to calibrate new design techniques, including the unsteady character of the flow. Many researchers have studied the flow field in the rotor-stator interaction zone, such as laser techniques such as Laser Doppler Velocimetry
(LDV) [1], and more recently, Particle Image Velocimetry (PIV) [2], but in order to get a better understanding of the flow behavior, it may be interesting to add measures pertaining to the pressure sensor for a complete analysis of the performance of the flow.

Main of the previous work, which has been done in this pump model, was devoted to perform velocity measurements. In order to get the complete flow characteristic information, it is also necessary to know outlet pressure values and the related global losses in one hand, and get more detailed pressure measurements just downstream the impeller at vaned diffuser inlet section in the other hand.

Because of real industrial pump dimensions, it is generally quite difficult to get such local 
information on local pressure at impeller outlet. One can usually measure only one static pressure on shroud and calculate the total head assuming that radial and tangential velocity values can be evaluated from flow rate and torque information respectively. In a specific pump model such as the present one, pump dimensions have been enlarged in order to be able to measure pressure for twenty three probe positions at diffuser inlet and outlet diffuser sections and inside the diffuser passage for five different flow rates.

To better optimize the design of the pump, numerical simulations of the internal flow, performed by the STAR-CCM+ software on eight different relative angular positions of the diffuser blades relative to the impeller blades (Frozen-rotor), are now available. Some geometric simplifications are used. For example, the flux leakage is often overlooked.

\section{Experiment}

The experimental work was carried out in ENSAM Lille. Tests were performed in the air with a centrifugal pump, on a test rig. A more detailed presentation of the test rig is proposed in $[3,4]$ and a sketch in figure 1 . The pump is equipped of the socalled SHF impeller coupled with a vaned diffuser shown in figures 2 and 3. The pump model has already been described in several papers as reference $[3,4,5,6,7]$, whose geometrical characteristics and operating conditions are reported in table 1 .

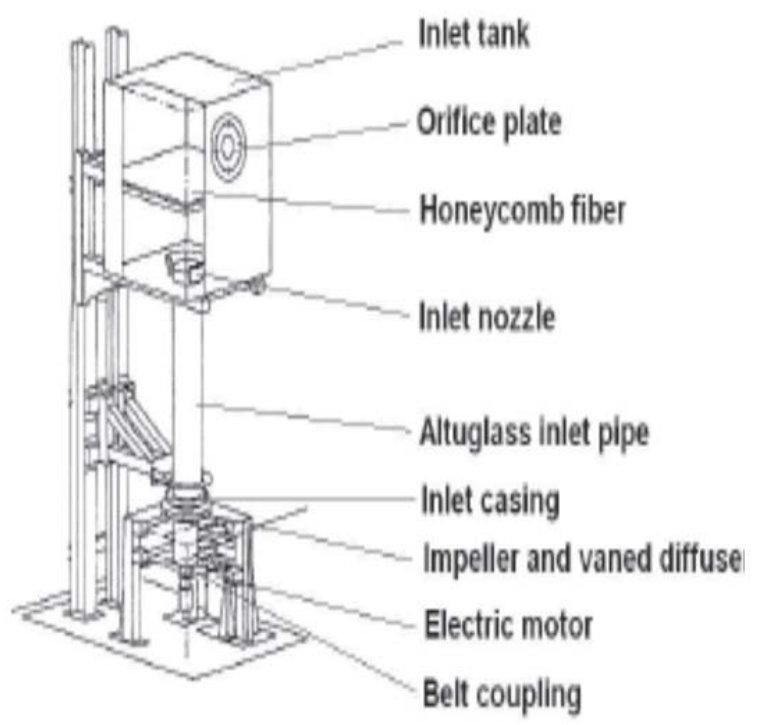

Fig.1 Sketch of the test rig
Table 1 Impeller and diffuser characteristics

\begin{tabular}{|l|l|}
\hline \multicolumn{2}{|l|}{ Entry duct } \\
\hline Diameter & $0.14113 \mathrm{~m}$ \\
\hline Length & $0.25 \mathrm{~m}$ \\
\hline Impeller & $\mathrm{R}_{1}=0.14113 \mathrm{~m}$ \\
\hline Inlet radius & $\mathrm{R}_{2}=0.2566 \mathrm{~m}$ \\
\hline Outlet radius & $\mathrm{B}_{2}=0.0385 \mathrm{~m}$ \\
\hline Outlet width & $\mathrm{Z}_{\mathrm{i}}=7$ \\
\hline Number of blades & $\beta_{2 \mathrm{a}}=22.30^{\circ}$ \\
\hline Outlet blade angle & $\mathrm{S}=0.009 \mathrm{~m}$ \\
\hline Mean blade thickness & $\mathrm{N}=1710 \mathrm{rpm}$ \\
\hline Design speed of rotation & $\mathrm{Q}_{\mathrm{d}}=0.3365 \mathrm{~m} 3 / \mathrm{s}$ \\
\hline Impeller design flow rate & \multicolumn{2}{|l|}{} \\
\hline Diffuser & $\mathrm{R}_{3}=0.2736 \mathrm{~m}$ \\
\hline Inlet radius & $\left(\mathrm{R}_{3}-\mathrm{R}_{2}\right) / \mathrm{R}_{2}=6.65 \%$ \\
\hline Relative radial gap & $\mathrm{R}_{4}=0.3978 \mathrm{~m}$ \\
\hline Outlet radius & $\mathrm{Z}_{\mathrm{d}}=8$ \\
\hline Number of vanes & $\begin{array}{l}\alpha 31=10.21^{\circ}(\text { at the blade } \\
\text { leading edge, } \mathrm{R}=0.27594 \mathrm{~m} \text { ) }\end{array}$ \\
\hline Inlet blade angle & $\begin{array}{l}\alpha 32=15.85^{\circ} \text { (in the center of } \\
\text { the inlet throat, } \mathrm{R}=0.29806 \mathrm{~m} \text { ) }\end{array}$ \\
\hline & $\begin{array}{l}\alpha 4=15.85^{\circ} \text { (in the center of } \\
\text { the inlet throat, } \mathrm{R}=0.37188 \mathrm{~m} \text { ) }\end{array}$ \\
\hline Outlet blade angle & $\mathrm{B}_{4}=0.04 \mathrm{~m}$ \\
\hline Height & $0.8 \mathrm{Q}_{\mathrm{d}}$ \\
\hline Design flow rate & \multicolumn{1}{|l|}{} \\
\hline
\end{tabular}

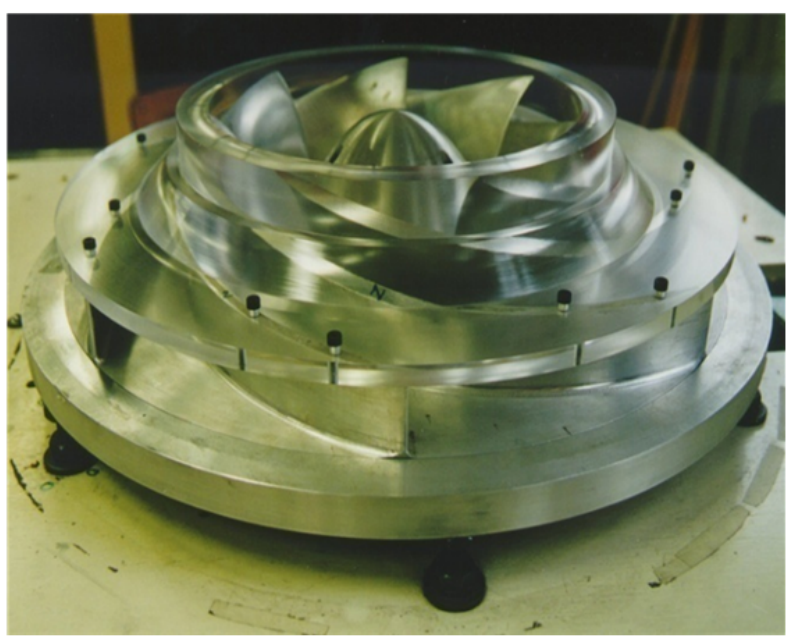

Fig.2 SHF Impeller with transparent 


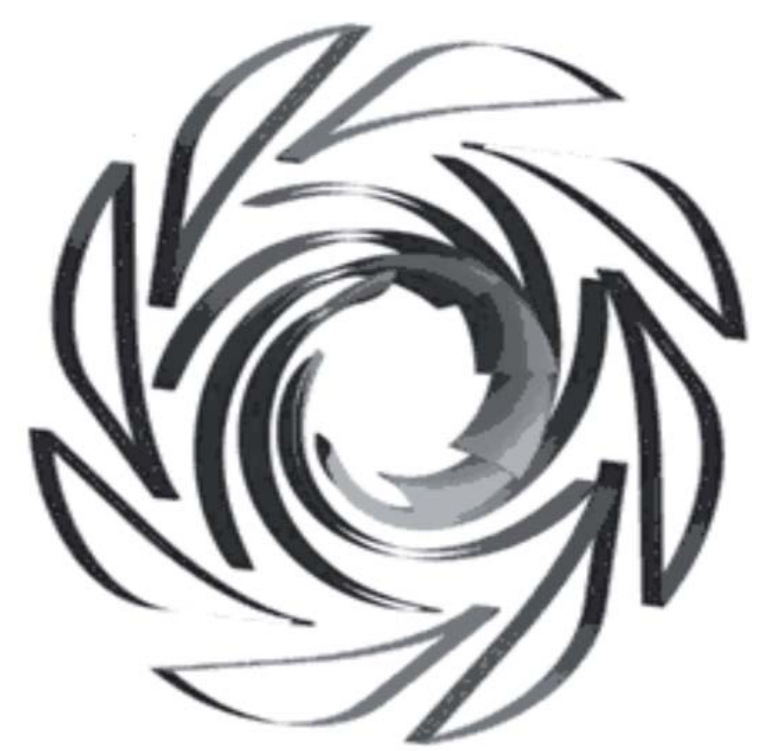

Fig.3 The SHF impeller coupled with the vaned diffuser.

\subsection{Three-hole probe measurements}

Several types of measurements have been carried out in the diffuser:

- The performance and the pressure rise of the diffuser were estimated by, three-hole probe measurements (figure 4) which holes are connected to a Honeywell type pressure tap device. The probe, with prior appropriate calibration permits to determine a total pressure, static pressure, absolute velocity and its two components in the radial and tangential direction. The size of the probe is assumed to be small enough not to disturb the flow within the diffuser circuit.

It has to be noticed that:

- A $0.3 \mathrm{~mm}$ gap does exist in impeller shroud tip inlet section and at impeller outlet both at the hub and shroud. In this respect, the impeller inlet mass flow rate has been evaluated taking into account this leakage flow.

- There is no volute downstream to the diffuser in order to focus the analysis on the impeller-diffuser interaction. Therefore, the flow at the outlet of diffuser discharges directly into surrounding atmosphere.

- The diffuser design flow rate is corresponding to $80 \%$ of the impeller design flow rate. This configuration has been chosen to obtain a better performance of the pump at partial flow rates.

For all campaigns, the impeller rotation speed was $1710 \mathrm{rpm}$, for five mass flow ratio $\left(\mathrm{Q} / \mathrm{Q}_{\mathrm{d}}=0.38\right.$,
$0.58,0.762,0.975$ and 1.14). The investigations will focus on the results obtained, at ten positions intermediate planes $\left(\mathrm{B}^{*}=0.1250 .20 .250 .3750 .5\right.$ 0.6250 .750 .8750 .9250 .975 ) between the hub and the shroud of the inter-blade channel diffuser (figure 5), for nine location positions in vaned diffuser inlet section.

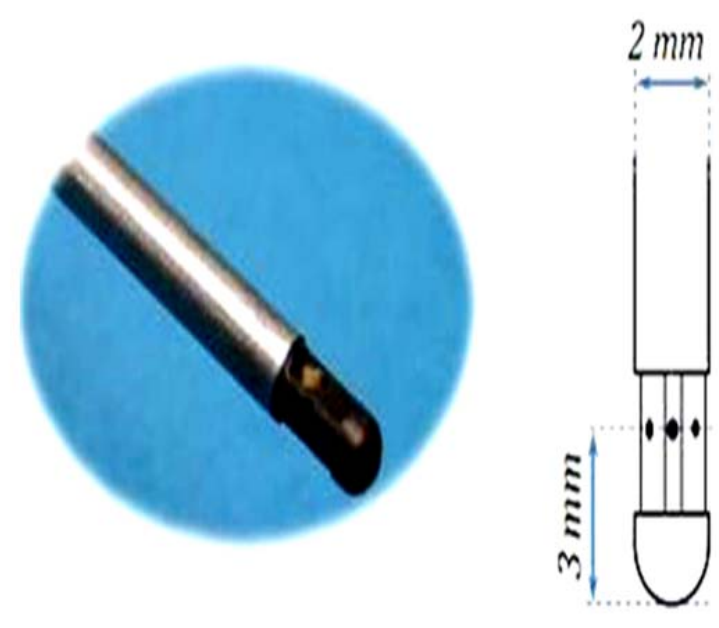

Fig.4 Picture and sketch of the three-hole pressure probe

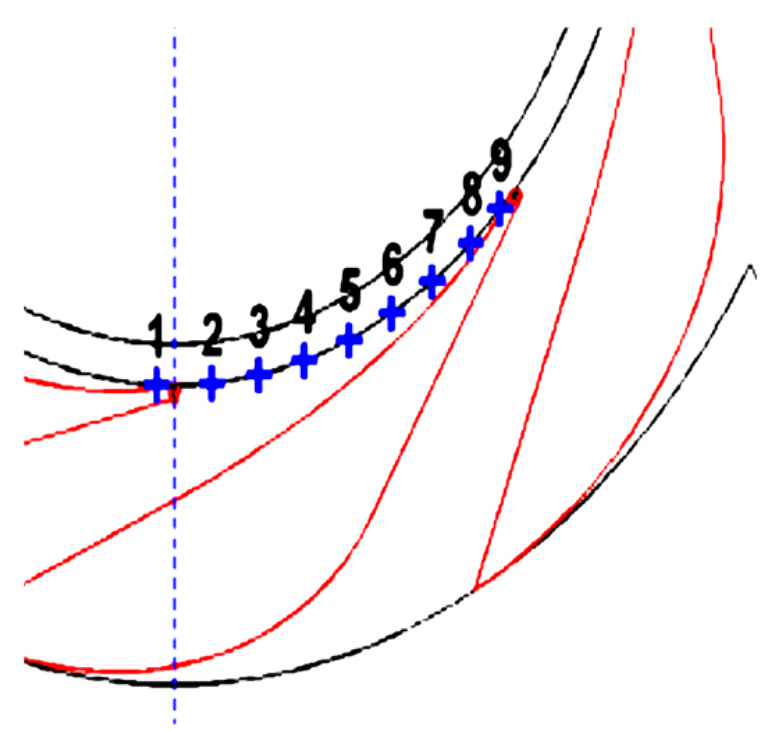

Fig.5 Pressure measurement probes locations traverse 


\subsection{Global performance}

The global performance in different part of the machine, is given by: $\mathbf{\Psi}=\boldsymbol{\Delta}_{\mathbf{s}} / \boldsymbol{\rho} \cdot \mathbf{U}_{\mathbf{2}}^{2}$

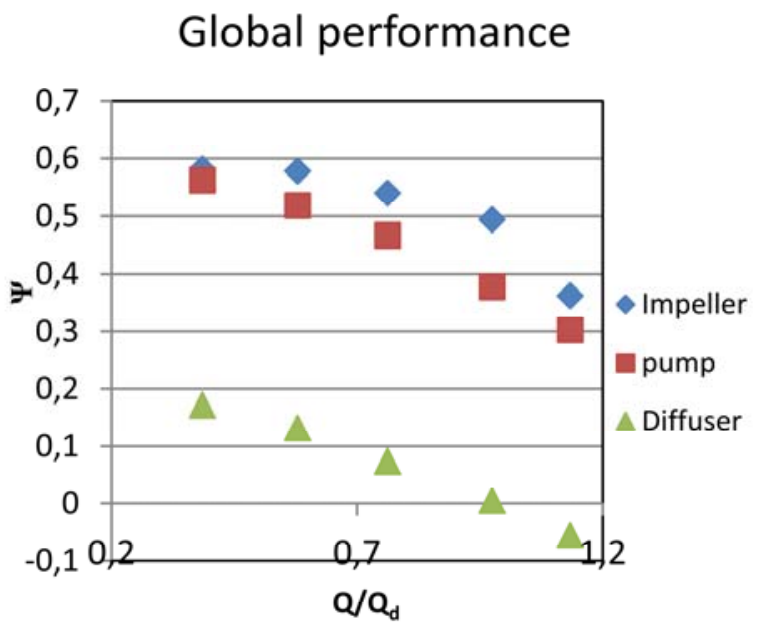

Fig.6 Global performance

Figure 6 shows the performance of the fan and its components. What is obvious in this figure is that the diffuser performance is very bad for $\mathrm{Q}>\mathrm{Q}_{\mathrm{d}}$, as the static pressure is decreasing which means there are great losses in it.

\section{Models description and computatio- -nal method}

\subsection{Mathematical models}

\subsubsection{Basic equations}

For three-dimensional incompressible, turbulent flow, the continuity and momentum equations can be written in the rotating coordinate system as follows:[8]

Continuity:

Momentum:

$$
\begin{aligned}
& \frac{\partial \rho \overrightarrow{\mathrm{W}}}{\partial \mathrm{t}}+\nabla(\rho \overrightarrow{\mathrm{W}} \wedge \overrightarrow{\mathrm{W}})=\nabla \cdot\left(-\mathrm{p} \delta+\mu_{\mathrm{eff}} \overrightarrow{\mathrm{D}}\right)-\overrightarrow{\mathrm{fC}^{\mathrm{C}}}-\overrightarrow{\mathrm{f}^{\mathrm{G}}} \\
& \text { with: } \quad \overrightarrow{\mathrm{D}}=\left(\nabla \overrightarrow{\mathrm{W}}+(\nabla \overrightarrow{\mathrm{W}})^{\mathrm{T}}\right) \\
& \qquad \overrightarrow{\mathrm{fC}^{\mathrm{C}}}=\rho 2 \vec{\Omega} \wedge \overrightarrow{\mathrm{W}} \\
& \overrightarrow{\mathrm{f}^{\mathrm{G}}}=\rho \vec{\Omega} \wedge(\vec{\Omega} \wedge \overrightarrow{\mathrm{r}})
\end{aligned}
$$

where $\mathrm{p}$ is the fluid pressure; $\rho$ is the density; $\delta$ is the identity matrix; $\vec{W}$ is the fluid velocity in the rotating system and $\vec{\Omega}$ is the rotation speed of the impeller, $r$ the location vector and $\vec{D}$ the strain tensor. The governing equations are solved in a rotating reference frame, so including $\overrightarrow{\mathrm{fC}}$ Coriolis and $\overrightarrow{\mathrm{f}} \overrightarrow{\mathrm{G}}$ centrifugal forces. The computational domain is a periodically symmetric section of the centrifugal impeller.

\subsection{2 k-E Turbulence model}

In Equation (3), $\mu_{\mathrm{eff}}$ is the effective viscosity coefficient, which equals the molecular viscosity coefficient, $\mu$, plus the turbulent eddy viscosity coefficient, $\mu_{\mathrm{t}}$ :

$$
\mu_{\mathrm{eff}}=\mu+\mu_{\mathrm{t}}
$$

The turbulent viscosity, $\mu_{\mathrm{t}}$, is modeled as the product of a turbulent velocity scale, $\mathrm{W}_{\mathrm{t}}$, and a turbulent length scale, $l_{t}$, as proposed by Kolmogorov [9]. Introducing a proportionality constant gives,

$$
\mu_{\mathrm{t}}=\rho \mathrm{C}_{\mu} \mathrm{l}_{\mathrm{t}} \mathrm{W}_{\mathrm{t}}
$$

Both equation models take the velocity scale, $W_{t}$, to be the square root of the turbulent kinetic energy:

$$
\mathrm{W}_{\mathrm{t}}=\sqrt{\mathrm{k}}
$$

The turbulent kinetic energy, $k$, is determined from the solution of a semi empirical transport equation. In the standard $k-\varepsilon$ two-equation model it is assumed that the length scale is a dissipation length scale, and when the turbulent dissipation scales are isotropic, Kolmogorov determined that

$$
\varepsilon=\frac{\mathrm{k}^{3 / 2}}{\mathrm{l}_{\mathrm{t}}}
$$

where $\varepsilon$ is the turbulent dissipation rate.

Therefore, the turbulence viscosity, $\mu_{\mathrm{t}}$, can be derived from Equations (8), (9), and (10) to link to the turbulence kinetic energy and dissipation via the relation

$$
\mu_{\mathrm{t}}=\mathrm{C}_{\mu} \rho \mathrm{k}^{2} / \varepsilon
$$

where $C_{\mu}$ is a constant. Its value is 0,09 .

The values of $k, \varepsilon$ come directly from the differential transport equations for the turbulence kinetic energy and turbulence dissipation rate:

$$
\begin{aligned}
& \frac{\partial \rho \mathrm{k}}{\partial \mathrm{t}}+\nabla(\rho W k)=\nabla\left(\Gamma_{\mathrm{k}}(\nabla k)\right)+\mathrm{P}_{\mathrm{k}}+\rho_{\mathrm{k}} \\
& \frac{\partial \rho \varepsilon}{\partial t}+\nabla(\rho W \varepsilon)=\nabla\left(\Gamma_{\varepsilon}(\nabla \varepsilon)\right)+\frac{\varepsilon}{k}\left(C_{\varepsilon_{1}} \mathrm{P}_{k}-\rho C_{\varepsilon_{2}} \varepsilon\right) \\
& \text { where the diffusion coefficients are given by }
\end{aligned}
$$

$$
\Gamma_{\mathrm{k}}=\mu+\frac{\mu_{\mathrm{t}}}{\sigma_{\mathrm{k}}} \quad \Gamma_{\varepsilon}=\mu+\frac{\mu_{\mathrm{t}}}{\sigma_{\varepsilon}}
$$

and $\mathrm{C}_{\varepsilon_{1}}=1,44 ; \mathrm{C}_{\varepsilon_{2}}=1,92 ; \sigma_{\mathrm{k}}=1,0$; and $\sigma_{\varepsilon}=1,3$ are constants. 
The $\mathrm{P}_{k}$ in Equations (12) and (13) is the turbulent kinetic energy production term, which for incompressible flow is

$$
\mathrm{P}_{k}=\mu_{t} \nabla \mathrm{W}\left(\nabla W+(\nabla W)^{T}\right)-\frac{2}{3} \nabla \mathrm{W}\left(\mu_{t} \nabla \mathrm{W}+\rho \mathrm{k}\right)
$$

Equations (1), (2), (8) and (9) form a closed set of nonlinear partial differential equations governing the fluid motion.

\subsubsection{Log-Law Wall Functions}

There are large gradients in the dependent variables near the wall. It is costly to fully resolve the solution in this near-wall region as the required number of nodes would be quite large. Thus a common approach known as "wall functions" is applied to model this region.

In the wall-function approach (Launder and Spalding[10]), the near-wall tangential velocity is related to the wall shear stress by means of a logarithmic relation, which can be written as follows:

Where

$$
\mathrm{u}^{+}=\frac{\mathrm{u}_{\mathrm{t}}}{\mathrm{u}_{\tau}}=\frac{1}{\mathrm{k}} \ln \left(\mathrm{y}^{+}\right)+\mathrm{C}
$$

$$
y^{+}=\frac{\rho y_{p} \mu_{\tau}}{\mu} \quad \text { and } \quad \mu_{\tau}=\sqrt{\frac{\tau_{\omega}}{\rho}}
$$

$\tau_{\omega}$ is the wall shear stress, $u_{t}$ is the known velocity tangent to the wall at a distance of $y_{p}$ from the wall $\mathrm{k}$ is the von Karman constant for smooth walls, $\mathrm{k}$ and $\mathrm{C}$ are constants, depending on wall roughness.

\subsubsection{The finite volume method}

The basic idea of the method presented here is to calculate approximately parameters of flow. Spatial discretization is performed on a computational domain, using finite volume techniques. The procedure followed is to divide the flow into disjoint cells by lines that define the boundary of each individual cell; the created pattern is called mesh. The flow is considered to be steady axis-symmetric.

- Basic Equations

The integration of the conservation equation on each volume $\Omega_{i}$ domain is given by:

$\int_{\Omega_{i}}\left(\frac{\partial \rho \emptyset}{\partial t}+\nabla(\rho W \emptyset)\right) d v=\int_{\Omega_{i}}\left(\nabla\left(\Gamma_{\varnothing} \nabla_{\varnothing}\right)+S_{\varnothing}\right) d v$

In order to solve the problem in each case, first step is to define a finite volume grid on a rectangular domain. The flow is divided into non overlapping control volumes by the lines that define the boundaries of the individual control volumes. The pattern created by the lines is called the computational grid or mesh. In figure 7 the control volumes are square, but this is not a requirement.
Two sets of grid lines can be identified: the grid lines that define the locations of the nodes, and the grid lines (dashed line) that define the control volume faces.
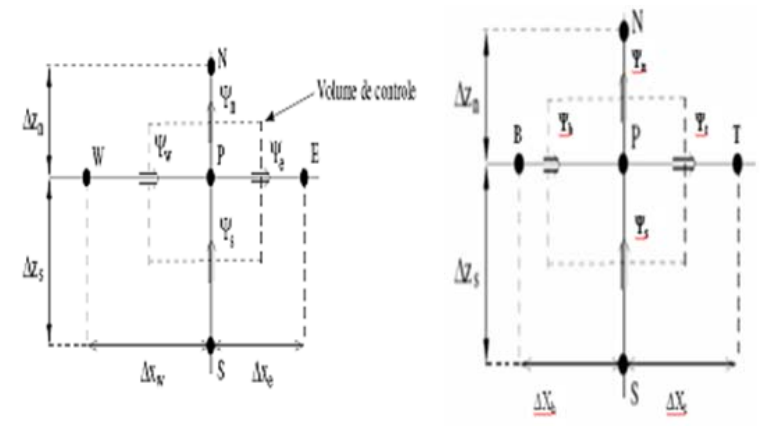

Fig.7 3D control Volume

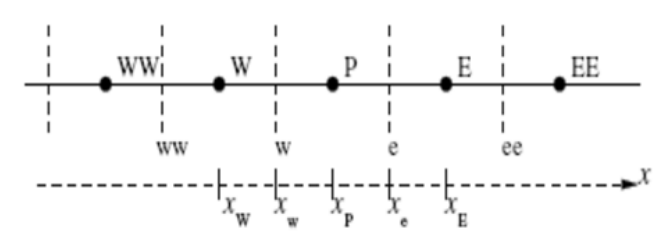

Fig.8 Computational grid

The finite volume method is used to transform (18) to a system of discrete equations for the nodal values of $\varnothing$. First (18) are integrated over the typical control volume depicted in figure 8 . This reduces each equation to one involving only first derivatives in space. Then these first derivatives are replaced with central difference approximations [11].

The conservation equation once implicitly discretized in time, is given by:

$$
\begin{aligned}
a_{p}^{n+1} \emptyset_{p}^{n+1}= & a_{w}^{n+1} \emptyset_{w}^{n+1}+a_{E}^{n+1} \emptyset_{E}^{n+1}+ \\
& a_{S}^{n+1} \emptyset_{S}^{n+1}+a_{N}^{n+1} \emptyset_{N}^{n+1}+ \\
& a_{B p}^{n+1} \emptyset_{B}^{n+1}+a_{T}^{n+1} \emptyset_{T}^{n+1}+ \\
& a_{p}^{n} \emptyset_{p}^{n}+b
\end{aligned}
$$

\subsection{Computational method}

Computations are performed using the STAR$\mathrm{CCM}+$ code. Three-dimensional, steady incompressible, Reynolds averaged Navier Stokes, including the centrifugal force source in the impeller are solved. The turbulence is simulated with the 
standard k- $\varepsilon$ model. Although grid size is not adequate to investigate local boundary layer variables, global ones are well captured. For such calculations, wall functions, based on the logarithmic law, have been used. flow problem was solved using the finite volume method.

\subsubsection{Pump geometry}

The pump is modeled as a set of three components: an entry duct, impeller, and vaned diffuser as shown in figure 9. Impeller rotates clockwise. The entry duct is chosen long enough to assume uniform upstream inlet flow conditions. No flow leakage occurring in the gaps between rotating and stationary parts was accounted for in the numerical study.

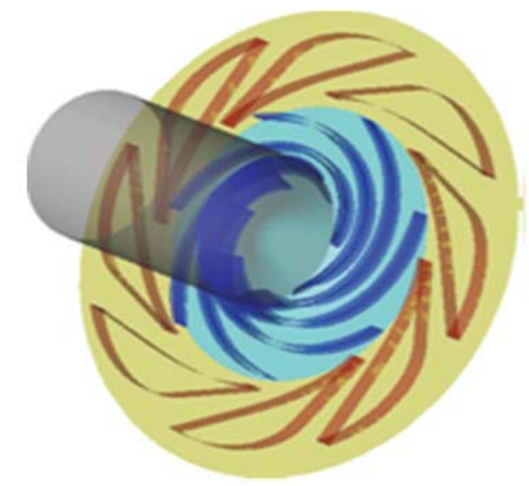

Fig.9 Pump geometry

\subsubsection{Test conditions}

The numerical simulation of the complete $360^{\circ}$ impeller and diffuser (figure 10) is carried out for different impeller angular positions relative to the diffuser vanes. This is equivalent to azimuthally positions equal to $n *=360 /\left(Z_{i} * Z_{d}\right)$ with $n=0,1,2,3$, 4, 5, 6 and 7.Line probes are plotted as defined in order to obtain all parameters (pressure, total pressure, radial, tangential and axial velocity and velocity magnitude) for different impeller angular positions relative to the diffuser vanes. In this paper, $\mathrm{P} 1$ is the only position presented and discussed.

The pump is tested at the same conditions as the experimental. It should be noted that the diffuser was designed to be fitted at $80 \%$ of the impeller design flow rate. This was intended to improve performance at low flow rates. The main features of the components and specifications of operating conditions are summarized in Table 1.

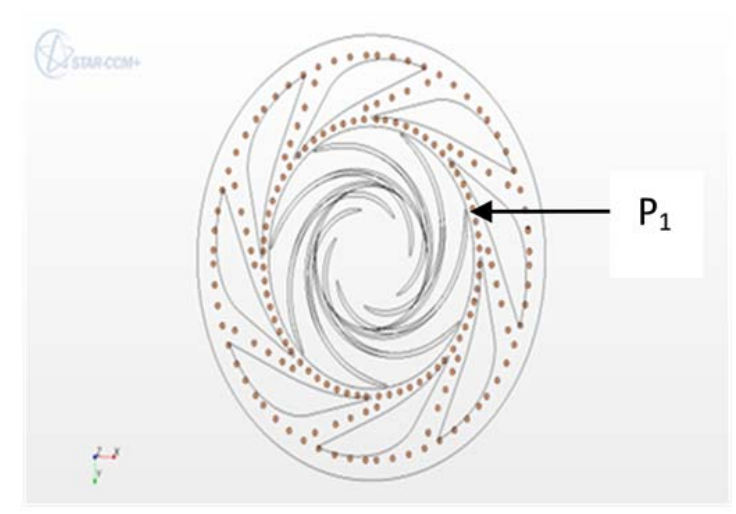

Fig.10 Probes for frozen rotor calculations.

\subsubsection{Mesh structure}

A polyhedral mesh with prism layers is used for all calculations (figure 11). Five prism layers for a total prism layer thickness of $1 \mathrm{~mm}$. The target size is 3 $\mathrm{mm}$ and the minimum size $0.5 \mathrm{~mm}$. The size of the grid is about $3.10^{6}$ cells. Mesh refinements are placed at particular locations like blade edges, walls, and interfaces.

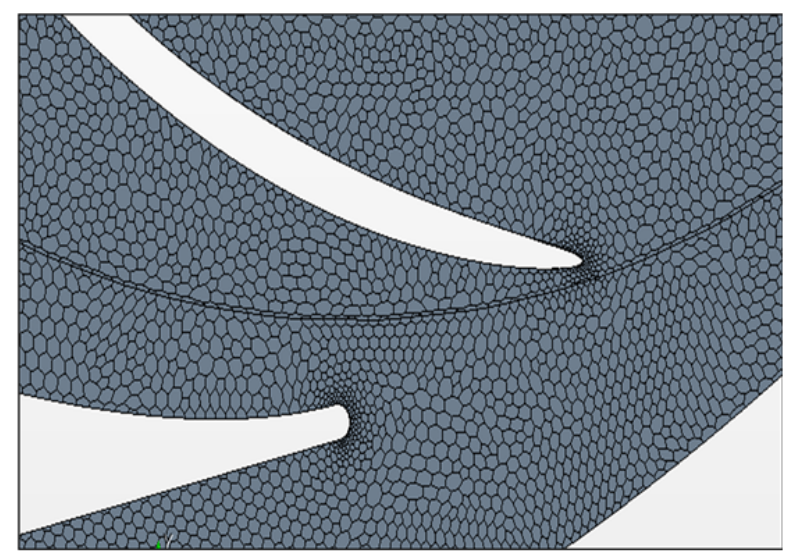

Fig.11 Local mesh between Impeller and diffuser

\subsubsection{Boundary conditions}

The calculation domain was divided into three zones: the inlet zone, the impeller zone and the diffuser zone. One rotational speed, $1710 \mathrm{rpm}$, was used in the computations. The modeled boundary conditions are the ones considered with more physical meaning for turbomachinery flow simulations. Mass flow rates obtained from the experimental data were prescribed at the inlet boundary and at the labyrinth close to the impeller inlet with stochastic fluctuations of the velocities with $5 \%$ free-stream turbulence intensity. The fluid 
air was considered incompressible at a constant temperature of $20^{\circ} \mathrm{C}$. As regards the exit boundary conditions, the atmospheric pressure was prescribed. This absolute condition is converted into the appropriate relative pressure in the rotating frame. The surfaces were supposed as adiabatic walls with a no-slip condition. An automatic near-wall treatment smoothly switched from a low-Reynolds number formulation to a wall function formulation.

\subsubsection{Post process}

The steady calculations were realized with the same mesh. The convergence criteria are less than 1. $e^{-4}$ Maximum values of $\mathrm{y}^{+}$are around 15, and mainly below 9 in the whole computational domain as can be seen in figure 12. The numerical results were elaborated both to be compared with the experimental data and to enrich the experimental results with information useful for phenomena interpretation.

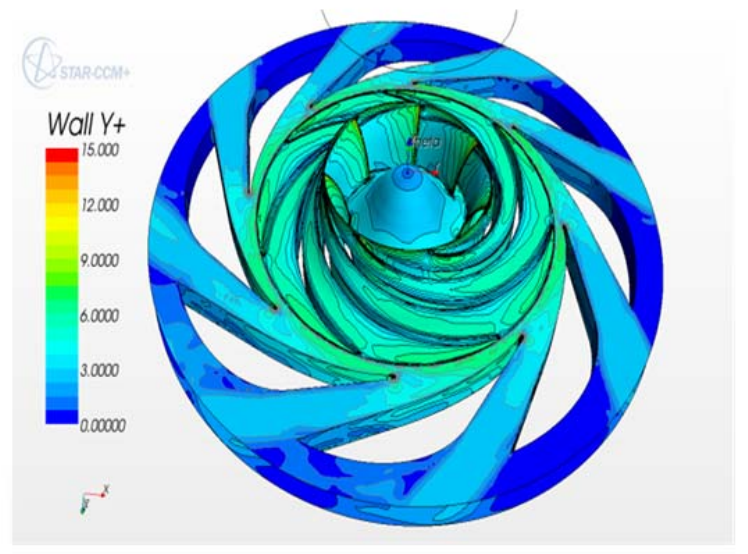

Fig. $12 \mathrm{y}^{+}$for steady calculations

\section{Experimental results within the impeller outlet, diffuser inlet.}

Because it is difficult to measure the total pressure within the gap between the impeller and diffuser of a centrifugal pump, pressure measurements are located at the inlet of the diffuser on the leading edge section allowing a complete survey of this section. The results of these measurements were obtained for several tangential positions between pressure faces and the depression from the diffuser vanes; these tangential positions are shown in figure 5 and are noted from 1 to 9 . Position 1 corresponds to a measurement of the pressure side of the diffuser blades; position 9 is located next to the depression of these panels.

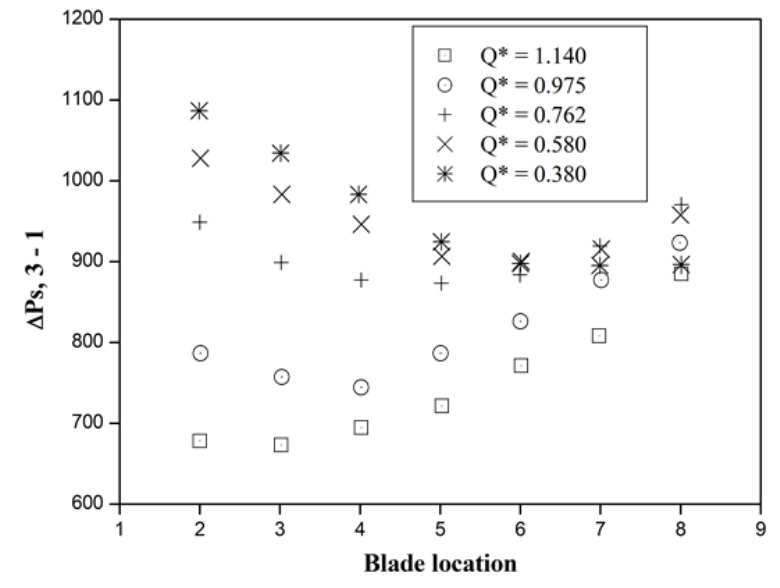

Fig.13. Inlet vaned diffuser blade to blade location

Figure 13 summarizes the results of the increase in static pressure $\Delta \mathrm{Ps}$ between the impeller inlet (section 1) and the diffuser inlet (section 3), obtained from the campaigns of measurement, whose values were extrapolated on the shroud side. It is thus possible to see that the gradient tangential of static pressure of the parietal extrapolation issued from surveys, are inverted according to the values of flow rates from one part and that the static pressure differences between the two faces of the diffuser vanes, which correspond to positions 1 and 9 , are the lowest valued dimensionless flow rate $\mathrm{Q} / \mathrm{Q}_{\mathrm{d}}=0.762$.

This value is close to the flow pattern of the diffuser and this confirms the existence of a low static pressure gradient corresponding to this flow.

We can also see that the values of static pressures vary little side depression blades when the exchange rate, while those corresponding to zones toward the pressure side are more sensitive to changes in flow. It is therefore not influenced by the presence of the direct edge regions of the blades of the diffuser that are most sensitive to variations in flow rate and therefore to variations in entrance angles along the diffuser inlet section.

Finally, the static pressure gradient is reversed when the flow is very large; this corresponds to negative angles of incidence at the leading edge of the blades. Face initially considered depressed nominal flow, is found naturally in pressure.

\section{Comparison between calculations and measurements of wall pressure.}

As presented below in figures 14, 15, 16 and 17 , the results of 3D RANS calculations from modeling " Frozen Rotor " and " Unsteady " category. They are always compared to the experimental results as 
regards to the changes in parietal static pressures. Both types of calculations have been performed for three flow rates of the four shown in figures

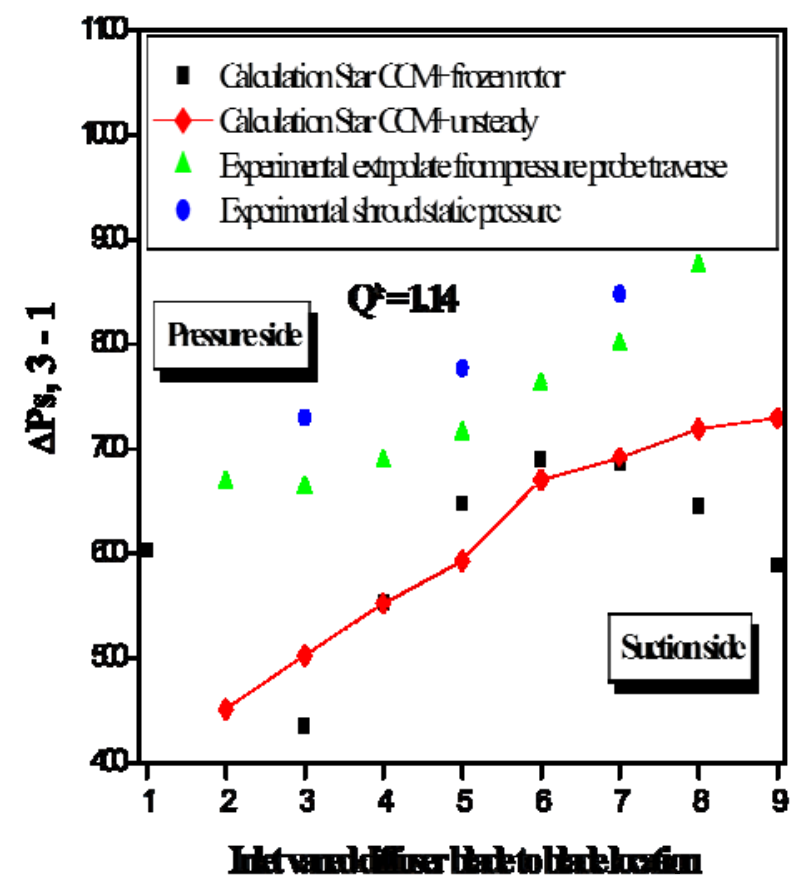

Fig.14 Distribution of tangential static pressure. Flow rate ratio $\mathrm{Q}^{*}=1,14$

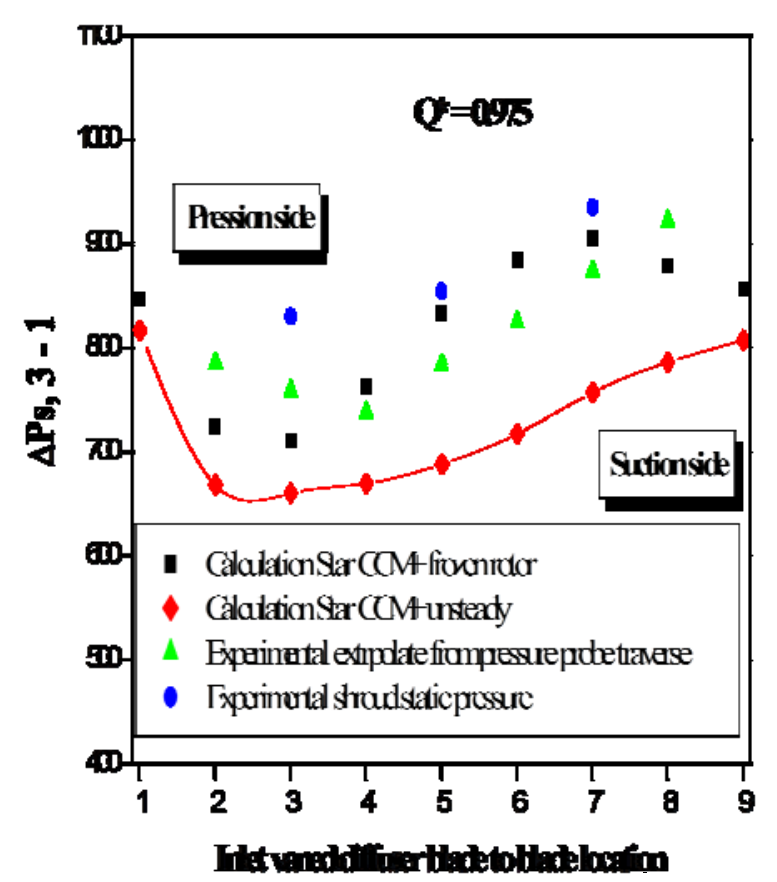

Fig.15 Distribution of tangential static pressure. Flow rate ratio $Q^{*}=0,975$

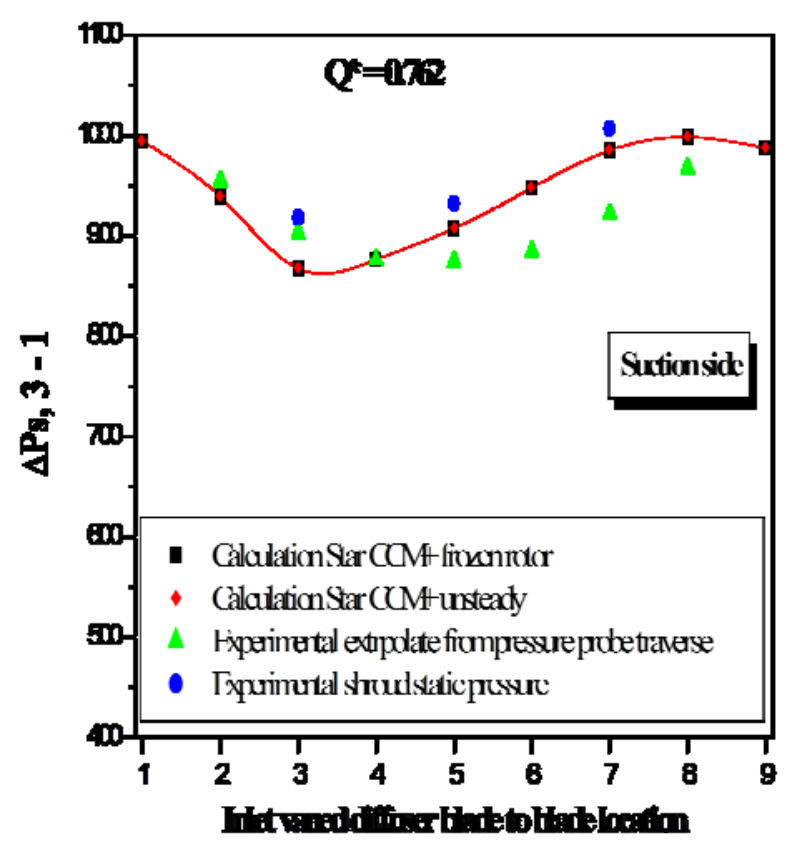

Fig.16 Distribution of tangential static pressure. Flow rate ratio $Q^{*}=0,762$

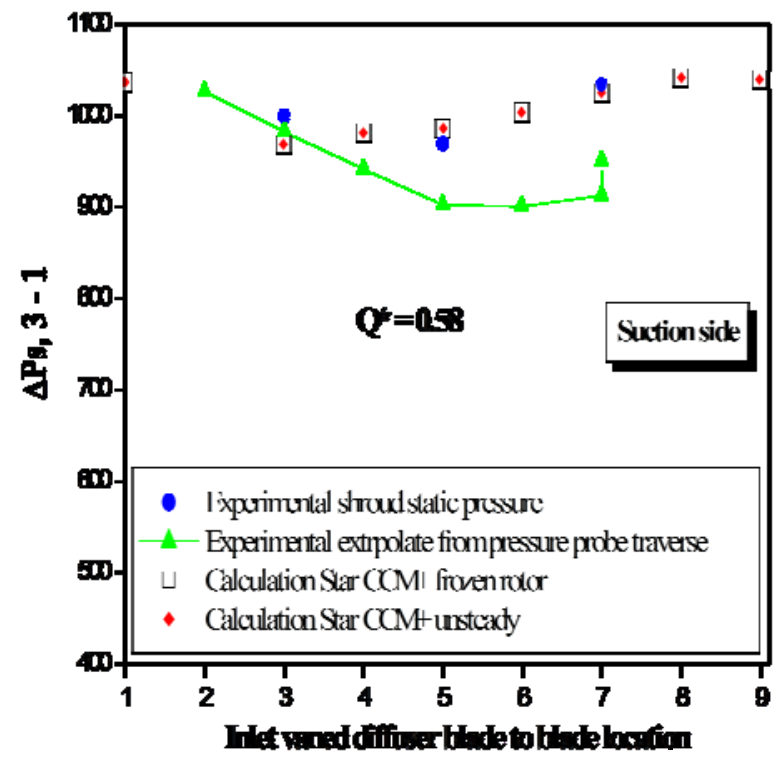

Fig.17 Distribution of tangential static pressure. Flow rate ratio $\mathrm{Q}^{*}=0,58$ 


\subsection{Comparison between measures of parietal pressure and the wall extrapolation sought from results.}

The experimental results obtained by these two measures show that differences exist between the levels of parietal static pressures which are generally stronger than the pressures obtained by extrapolation surveys. However, the tangential gradients are well respected. Moreover, differences in pressure level are all lower than the rate seen decreases. The differences between the two measurement techniques can be explained by blocking the effects related to the presence of the scanning probe in the flow itself.

\subsection{Comparison between the two numerical approaches}

Calculation results between the approach in" frozen" rotor and the completely unsteady approach are very close to each other, which shows that the values and tangential gradients of the static pressure at the inlet diffuser result essentially from the effect of the leading edges and the average impact of the flow. The unsteady nature thereof does not therefore affect, at this level, the pressure gradients.

\subsection{Comparison between measurements and simulation results}

We can see that the results are very close to the nominal flow of the diffuser, that is to say, for $\mathrm{Q}^{*}=$ 0.762 , figure 16 . For higher speeds, the interaction effects between the impeller and the diffuser vanes have an influence on the results which can be seen in figures 14 and 15.

These results should be analyzed in detail, especially in terms of evaluation of the mean values extracted from unsteady calculation techniques, because the values are consistently lower than those of stationary calculations and measurements, which, if these results were considered valid, would tend to believe that the losses in the smooth part of the diffuser between the trailing edge of the impeller and the diffuser inlet are higher than those obtained by the stationary calculation.

\section{Conclusion}

Several sources of interpretation of results should be compared to the local results obtained in this work. Indeed, we have in addition to the results taken of the parietal static pressure measuring gear module and direction from surveys and other measurements that are non-intrusive optical techniques of type PIV. These analyzes permit to link the results obtained from different measurement techniques and modelling should help to better analyze complex flows associated with interaction effects between the impeller and the diffuser outside the adaptation points of the centrifugal machines of this category.

\section{References:}

[1] K. Eisele, Z. Zhang, M. V. Casey, J. Gülich, and A. Schachenmann, Flow analysis in a pump diffusor part 1: LDA and PTV measurements of the unsteady flow, Journal of FluidsEngineering, vol. 119, no. 4, 1997, pp. 968-977.

[2] A. Dazin, O. Coutier-Delgosha, P. Dupont, S. Coudert, G. caignaert, G. Bois, Rotating stall in the vaneless of a radial flow pump, Procceedings of the 8th international Symposium on Experimental and Computational Aerothermo-dynamics of Internal Flows, Lyon, paper, 2007, July ISAIF8-00102.

[3] G. Wuibaut, G. Bois, M. El Hajem, A. Akhras, J.Y. Champagne, Optical PIV and LDV Comparisons of Internal Flow Investigations in SHF Impeller, Int. J. of Rotating Machinery, 2006, 1-9.

[4] G. Cavazzini, P. Dupont, G. Pavesi, A. Dazin, G. Bois, A. Atif, P. Cherdieu, Analysis of unsteady flow velocity fields inside the impeller of a radial flow pump: PIV measurements and numerical calculation comparisons, Proc. of ASME-JSME-HSME Joint fluids engineering conference, July 24-29, 2011, Hamamatsu, Japan.

[5] G. Cavazzini, G. Pavesi, G. Ardizzon, P. Dupont, S. Coudert, G. Caignaert, G. Bois, Analysis of the rotor-stator interaction in a radial flow pump Houille blanche, revue internationale de l'eau, 2011.

[6] G. Pavesi, G. Cavazzini, P. Dupont, S. Coudert, G. Caignaert, and G. Bois, Analysis of rotorstator interactions effects within the vaned diffusor of a radial flow pump, in Proceedings of the 23rd IAHR Symposium on Hydraulic Machinery and Systems, 2006, Yokohama, Japan.

[7] G. Wuibaut, P. Dupont, G. Caignaert, M. Stanislas, Experimental analysis of velocities in the outlet part of a radial flow pump impeller and the vaneless diffusor using particle image velocimetry, XX IAHR Symposium, Charlotte, 2000, USA.

[8] Bayeul-Laine AC, Dupont P, Miccoli L, Gavazzini G, Dazin A, Pavesi G, Bois, Fluid 
leakage effect on analysis of a vaned diffuser of SHF pump 15th International Symposium on Transport Phenomena and Dynamic of Rotating Machinery ISROMAC-15, 2014, (Honolulu, USA).

[9] Bayeul-Laine AC, Dupont P, Gavazzini G, Pavesi G, Dazin A, Cherdieu P, Bois G, Roussette O, Comparisons RANS and URANS numerical results with experiments in a vaned diffuser of a centrifugal pump La Houille blanche, 2015, pp 108-116.

[10] Andrei Giniatoulline, Mathematical Modeling of the Rotating Stratified Fluid in a Vicinity of the bottom of the Ocean International Journal of Mechanics, Volume 13, 2019, pp. 149-155.

[11] Kolmogorov, A. N., Local structure of turbulence in incompressible viscous fluid for very largeReynolds number, Doklady Akademyia Nauk SSSR, Vol. 30, 1941, pp. 913.

[12] Launder, B.E., and Spalding, D.B., The numerical computation of turbulent flows, Complete Methods of Applied Mechanical Engineering, Vol. 3, 1974, pp. 269-289.

[13] H. Versteeg and W. Malalasekera, An introduction to computational fluid dynamics, the finite volume method, Prentice Hall, 2007. 